\title{
W Mass and Width Measurements at the Tevatron
}

\author{
Sarah Malik \\ University College London - Department of Physics and Astronomy \\ Gower Street, London, WC1E 6BT, U.K.
}

\begin{abstract}
A measurement of the $\mathrm{W}$ boson mass $\left(M_{W}\right)$ and width $\left(\Gamma_{W}\right)$ using 200 and $350 \mathrm{pb}^{-1}$ of CDF Run II data respectively is presented. The measurements were performed in both the electron and muon channels. The $W$ mass is obtained by fitting the peak of the $W$ transverse mass distribution, whereas the width is extracted by fitting the tail of the distribution. We measure $M_{W}=80413 \pm 48 \mathrm{MeV}$ and $\Gamma_{W}=2032 \pm 71 \mathrm{MeV}$ both of which represent the single most precise measurements of these quantities to date.
\end{abstract}

\section{Introduction}

The mass of the $W$ boson receives radiative corrections from loops of virtual particles. The most dominant contributions are from the Higgs boson loop and the top-bottom loop where the Higgs boson contribution is proportional to the logarithm of its mass $\left(\mathrm{M}_{H}\right)$. A precision measurement of the $W$ mass therefore allows us to place an indirect constraint on $\mathrm{M}_{H}$. The width of the $\mathrm{W}$ boson is predicted with high precision within the Standard Model (SM), its measurement provides a valuable test of the SM prediction.

$W$ bosons are produced in proton-antiproton collisions at the Tevatron, predominantly via valence quark anti-quark annihilation. Events are selected where the $\mathrm{W}$ boson decays leptonically to $e \nu$ or $\mu \nu$ as these decay modes provide relatively clean signatures for detection. The invariant mass of the $\mathrm{W}$ is difficult to reconstruct, since a large fraction of longitudinal information is lost as fragments of the $q \bar{q}$ collision escape down the beam pipe. Transverse quantities are therefore used for the measurements, in particular the transverse mass, $\mathrm{M}_{T}$, which is defined as:

$$
M_{T}=\sqrt{2 p_{T}^{l} p_{T}^{\nu}\left(1-\cos \phi_{l \nu}\right)},
$$

where $\mathrm{p}_{T}^{l}$ is the transverse momentum of the charged lepton, $\mathrm{p}_{T}^{\nu}$ is the transverse momentum of the neutrino and $\phi_{l \nu}$ is the azimuthal angle between the charged lepton and the neutrino. The $\mathrm{W}$ mass is extracted by fitting the peak of the $\mathrm{M}_{T}$ distribution, the region $65-90 \mathrm{GeV}$. The width is obtained by fitting the tail of the distribution (90-200 GeV) and exploiting the slower fall-off of the Breit-Wigner lineshape compared to the detector resolution.

A dedicated, fast Monte Carlo simulation is used to generate the $\mathrm{M}_{T}$ distribution used in the fits. Parton Distribution Functions (PDFs) are provided by the CTEQ6M [6] set. The $\mathrm{W}$ is produced with a non-zero transverse momentum, and the shape of the $p_{T}$ spectrum is taken from RESBOS [3]. All final state QED radiation is simulated with the Berends and Kleiss [4] program for the width and WGRAD [5] for the mass.

\section{Lepton Momentum Scale and Resolution}

One of the key aspects of the measurement is an accurate determination of the lepton momentum. The momentum of the lepton is measured in the Central Outer Tracker(COT), a cylindrical drift chamber placed in a magnetic field of $1.4 \mathrm{~T}$. Charged particles ionise 
atoms of the gas in the chamber producing a track where the momentum of the lepton can be inferred from the curvature of the track. The momentum scale and resolution of the COT is calibrated using the control samples, $\mathrm{J} / \psi \rightarrow \mu \mu, \Upsilon(1 S) \rightarrow \mu \mu$ and $Z \rightarrow \mu \mu$. The invariant mass of the dimuon pair is measured and compared to the world average mass of the reconstructed particle, in order to calibrate the detector.

\section{Lepton Energy Scale and Resolution}

The energy of the lepton is measured in the Central Electromagnetic Calorimeter (CEM). The CEM is calibrated by using $\mathrm{E} / \mathrm{p}$, the ratio of the energy measured in the calorimeter to the momentum measured in the COT. By using this quantity, the already well calibrated momentum measurement can be used to calibrate the calorimeter. The energy scale obtained from this method is then cross-checked by using the resonance peak of the $Z \rightarrow e e$ sample. The invariant mass of the dielectron pair as measured in the calorimeter is compared to the world average mass of the $\mathrm{Z}$ boson.

\section{Hadronic Recoil Calibration}

The neutrino is not detected in the CDF detector, its transverse momentum can be inferred from the missing transverse energy $\left(E_{T}\right)$ in the detector. This is obtained by summing the energy of all calorimeter towers excluding those containing or neighbouring the electron. This is what is referred to as the recoil, and it is denoted by $\vec{U}$. The $\mathbb{E}_{T}$ can then be defined in terms of the recoil as $-\left(\vec{U}+\vec{p}_{T}^{\vec{l}}\right)$.

The recoil receives contributions from three main sources. When the $\mathrm{W}$ boson is produced, it recoils against initial state gluon radiation from the incoming quarks giving it a net non-zero transverse momentum. Gluon radiation forms hadronic jets that end up in the calorimeter. Other processes coinciding with $\mathrm{W}$ boson production(underlying event) also contribute to the recoil as well as final state photon radiation from the charged lepton which is not emitted collinear with the lepton.

The recoil can be resolved into two components, $U_{1}$ which is parallel to the direction of the $p_{T}$ of the $\mathrm{Z}$ and $\mathrm{U}_{2}$ which is perpendicular to it. $\mathrm{U}_{1}$ is largely boson $p_{T}$ dependent and $\mathrm{U}_{2}$ is mostly underlying event dependent. This enables a parametrisation of the recoil in terms of these components. Parameters of the model are determined by fitting to $Z \rightarrow l l$ data and minimum-bias data.

\section{Backgrounds}

The $\mathrm{W}$ event sample is contaminated by backgrounds arising from several sources. These can be divided into two types, electroweak and non-electroweak backgrounds.

Electroweak backgrounds consist of $Z \rightarrow l l$ events which can fake a $\mathrm{W}$ event if one of the leptons is not reconstructed and $W \rightarrow \tau \nu$ where the $\tau$ decays to an electron or muon. Non-electroweak backgrounds consist of multi-jet events and in the muon channel, kaons and pions decaying in the COT.

$\mathrm{W}$ bosons decaying to hadrons can fake $W \rightarrow l \nu$, if one of the jets fakes a lepton and the other is mis-measured producing a false $E_{T}$. In the muon channel, kaons and pions that decay in the drift chamber can produce a track that contains a kink and this can be 


\begin{tabular}{|l|c|c|c|}
\hline$\Delta M_{W}[\mathrm{MeV}]$ & $\mathrm{e}$ & $\mu$ & $\mathrm{C}$ \\
\hline Lepton Scale & 30 & 17 & 17 \\
Lepton Resolution & 9 & 3 & 0 \\
Recoil Scale & 9 & 9 & 9 \\
Recoil Resolution & 7 & 7 & 7 \\
Lepton ID & 3 & 1 & 0 \\
Lepton Removal & 8 & 5 & 5 \\
Backgrounds & 8 & 9 & 0 \\
$p_{T}(W)$ & 3 & 3 & 3 \\
PDF & 11 & 11 & 11 \\
QED & 11 & 12 & 11 \\
\hline Total Systematic & 39 & 27 & 26 \\
\hline Statistical & 48 & 54 & 0 \\
\hline Total & 62 & 60 & 26 \\
\hline
\end{tabular}

\begin{tabular}{|l|c|c|c|}
\hline$\Delta \Gamma_{W}[\mathrm{MeV}]$ & $\mathrm{e}$ & $\mu$ & $\mathrm{C}$ \\
\hline Lepton Scale & 21 & 17 & 12 \\
Lepton Resolution & 31 & 26 & 0 \\
Simulation & 13 & 0 & 0 \\
Recoil & 54 & 49 & 0 \\
Lepton ID & 10 & 7 & 0 \\
Backgrounds & 32 & 33 & 0 \\
$p_{T}(W)$ & 7 & 7 & 7 \\
$\mathrm{PDF}$ & 16 & 17 & 16 \\
$\mathrm{QED}$ & 8 & 1 & 1 \\
$M_{W}$ & 9 & 9 & 9 \\
\hline Total Systematic & 78 & 70 & 23 \\
\hline Statistical & 60 & 67 & 0 \\
\hline Total & 98 & 97 & 23 \\
\hline
\end{tabular}

Table 1: Systematic and statistical uncertainties for the $\mathrm{W}$ mass (left) and width (right). The last column denotes the uncertainties that are common between the electron and the muon channels.

falsely reconstructed as a track with a high $p_{T}$. The amount and shape of background contamination in the signal sample is estimated using data for non-electroweak background and Pythia [2] Monte Carlo for electroweak background. Backgrounds are added to the Monte Carlo templates.

\section{Results}

The systematic and statistical uncertainties for $M_{W}$ and $\Gamma_{W}$ are summarised in Table1. $M_{W}$ was obtained by fitting the three kinematic distributions $m_{T}, p_{T}^{l}$ and $p_{T}^{\nu}$. The combined fitted value obtained is $M_{W}=80413 \pm 48 \mathrm{MeV}$, which is the world's most precise single measurement of this quantity. The result increases the world average central value by $6 \mathrm{MeV}$ and reduces the uncertainty by $15 \%$. Figure 1 shows the $M_{T}$ fits for $M_{W}$ in the electron and muon channels.

Figure 2 shows the $M_{T}$ fits for $\Gamma_{W}$ in the electron and muon decay channels. The combined fitted value obtained is $\Gamma_{W}=2032 \pm 71 \mathrm{MeV}$, also the world's most precise single direct measurement of this quantity. This result reduces the world average central value by $44 \mathrm{MeV}$ and the uncertainty by $22 \%$ and is in good agreement with the Standard Model prediction. 

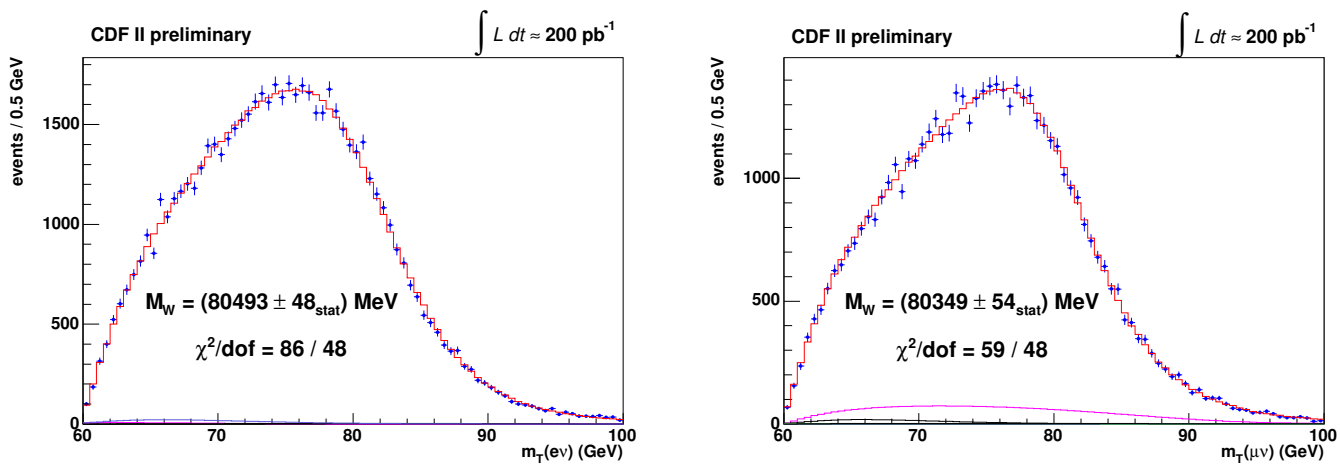

Figure 1: Transverse mass fits for $M_{W}$ in $W \rightarrow e \nu$ (left) and $W \rightarrow \mu \nu$ (right)
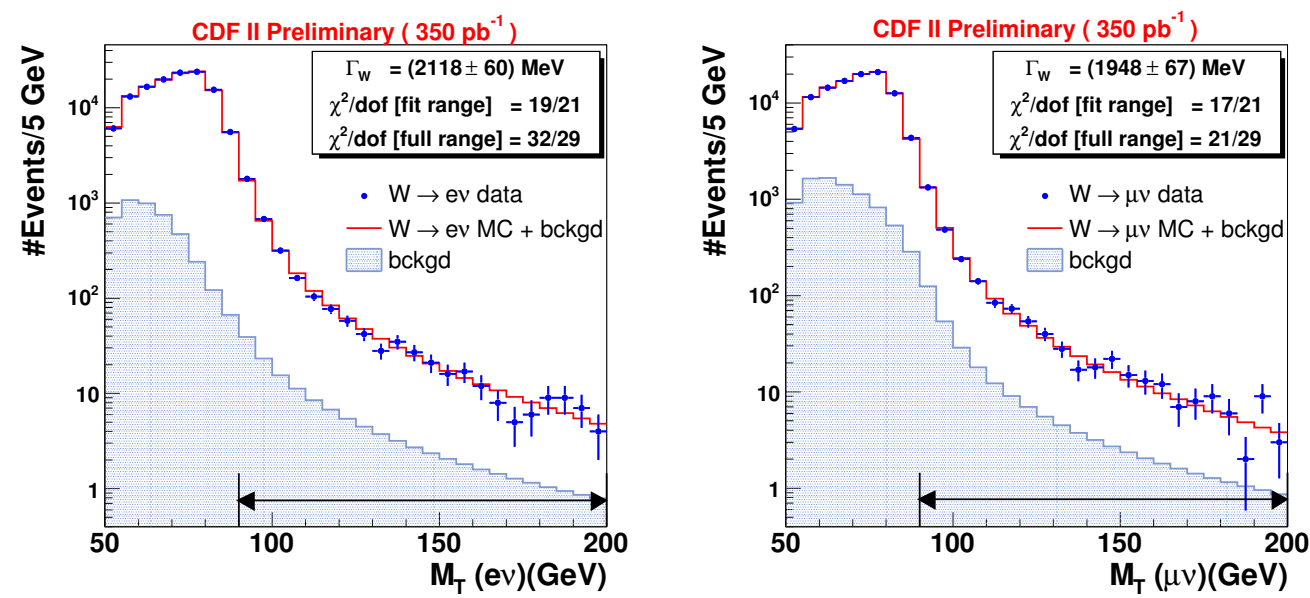

Figure 2: Transverse mass fits for $\Gamma_{W}$ in $W \rightarrow e \nu$ (left) and $W \rightarrow \mu \nu$ (right)

\section{References}

[1] Slides: http://indico. cern. ch/contributionDisplay . py? contribId=105\&sessionId=9\&conf Id=9499

[2] T. Sjostrand, P. Eden, C. Friberg, L. Lonnblad, G. Miu, S. Mrenna and E. Norrbin, Comput. Phys. Commun. 135 (2001) 238.

[3] C. Balazs, C. P. Yuan, Phys. Rev. D 56, (1997) 5558.

[4] F. A. Berends, R. Kleiss, J. P. Revol and J. P. Vialle, Z. Phys. C 27 (1985) 155; F. A. Berends and R. Kleiss, Z. Phys. C 27 (1985) 365.

[5] U. Baur, S. Keller and D. Wackeroth, Phys. Rev. D 59, (1998) 013002.

[6] J. Pumplin, D. R. Stump, J. Huston, H. L. Lai, P. Nadolsky and W. K. Tung, JHEP 0207 (2002) 012. 